\title{
Evaluación positiva de medicamentos: 1.er trimestre 2011
}

\author{
González Alfonso M. ${ }^{1}$, Prats Olivan P. ${ }^{2}$, García Luque A. ${ }^{3}$, Puerro Vicente M. ${ }^{4}$
}

Sanid. mil. 2011; 67 (2): 105-107; ISSN: 1887-8571

\section{RESUMEN}

Se reseñan los medicamentos ya evaluados por la Agencia Española de Medicamentos y Productos Sanitarios hechos públicos en el primer trimestre de 2011, y considerados de mayor interés para el profesional sanitario en el ámbito hospitalario. Se trata de opiniones técnicas positivas que son previas a la autorización y puesta en el mercado del medicamento.

\section{Positive assessment of drugs: $1^{\text {st }}$ quarter 2011}

SUMMARY

The drugs assessed by the Spanish Agency for Medicines and Health Products made public in the first quarter of 2011, and considered of interest in hospital healthcare professional, are reviewed. These are positive technical reports prior to the authorization and placing on the market of the product.

\section{APIXABAN $\left(\text { Eliquis }^{\circledR}\right)^{1,2}$}

El apixaban es un inhibidor reversible directo y selectivo del factor Xa inhibiendo el factor Xa libre, el unido al trombo y la actividad de protrombinasa, causando la inhibición indirecta de la agregación plaquetaria inducida por la trombina.

La indicación aprobada es la prevención de tromboembolismo en pacientes adultos que han sido sometidos a cirugía electiva de reemplazo de cadera o rodilla. En los ensayos clínicos, previos a su autorización, ha mostrado tener propiedades anticoagulantes y actividad antitrombótica en la prevención de tromboembolismos venosos, presentando como reacciones adversas más frecuentemente observadas durante el desarrollo clínico, anemia, hemorragia, confusión y náuseas.

\section{BROMFENACO SÓDICO $\left(\text { Yellox }^{\circledR}\right)^{1,3}$}

El principio activo de este fármaco es bromfenaco, un antiinflamatorio no esteroideo que actúa bloqueando la síntesis de prostaglandinas, fundamentalmente mediante la inhibición de la ciclooxigenasa 2 (COX-2).

La indicación aprobada del bromfenaco sódico es el tratamiento de la inflamación ocular postoperatoria tras extracción de catarata en adultos. En los ensayos clínicos se ha mostrado eficaz en el tratamiento de la inflamación ocular postoperatoria en pacientes sometidos a extracción de cataratas con implantación de lentes intraoculares en la cámara posterior.

${ }^{1}$ Tcol. Farmacéutico. Servicio de Farmacia Hospitalaria.

${ }^{2}$ Cap. Farmacéutico. Servicio de Farmacia Hospitalaria.

${ }^{3}$ Cte. Médico. Servicio de Farmacología Clínica.

${ }^{4}$ Tcol. Médico. Servicio de Farmacología Clínica.

Hospital Central de la Defensa Gómez Ulla. Madrid. España.

Dirección para correspondencia: Servicio de Farmacia Hospitalaria. Hospital Central de la Defensa Gómez Ulla. 28047 Madrid. España. mgonalf@oc.mde.es

Recibido: 13 de mayo de 2011

Aceptado: 24 de mayo de 2011
Las reacciones adversas más frecuentes observadas durante el desarrollo clínico fueron sensaciones anormales en el ojo, erosión corneal (leve o moderada), prurito ocular, dolor ocular y rojez ocular.

\section{CABAZITAXEL/Jevtana $\left.{ }^{\circledR}\right)^{4}$}

Cabazitaxel, es un agente antineoplásico que se une a la tubulina y promueve el ensamblaje de la tubulina a los microtúbulos al mismo tiempo que inhibe su desensamblado, esto conduce a la estabilización de los microtúbulos lo que se traduce en la inhibición de las funciones de mitosis e interfase celular.

Está indicado, en combinación con prednisona o prednisolona, para el tratamiento de cáncer de próstata metastático hormono-resistente en pacientes previamente tratados con quimioterapia con docetaxel.

Las reacciones adversas más comunes observadas durante la fase de investigación clínica fueron diarrea, fatiga, náuseas, vómitos y neutropenia.

\section{CLORURO DE METILTIONINIO ${\left.\text { (Cloruro de metiltioninio Proveblue }{ }^{\circledR}\right)^{6}}^{6}$}

El cloruro de metiltioninio es una sustancia que acelera la conversión de metahemoglobina en hemoglobina, se utiliza en la práctica clínica para tratar la metahemoglobinemia inducida por medicamentos o productos químicos. Este uso está debidamente documentado y la experiencia clínica ha demostrado la eficacia del cloruro de metiltioninio para revertir la mayoría de los casos de metahemoglobinemia.

Su indicación aprobada es el tratamiento sintomático de la metahemoglobinemia aguda inducida por medicamentos o productos químicos en adultos, niños y adolescentes (edades comprendidas entre 0 y 17 años).

Las reacciones adversas más comunes son náusea, dolor abdominal y torácico, cefalea, mareo, temblor, ansiedad, confusión, disnea, taquicardia, hipertensión, formación de metahemoglobinemia e hiperhidrosis. 


\section{ERIBULINA $\left(\text { Halaven }^{\circledR}\right)^{5}$}

El principio activo es mesilato de eribulina, que ejerce su efecto mediante un mecanismo antimitótico basado en la tubulina produciendo un bloqueo del ciclo celular G2/M que termina en muerte celular apoptótica tras un bloqueo mitótico prolongado.

La indicación aprobada es el tratamiento de pacientes con cáncer de mama localmente avanzado o metastático con progreso de la enfermedad en estadios avanzados después de al menos dos regímenes quimioterápicos.. El tratamiento previo, a menos que los pacientes no fueran adecuados para estos medicamentos, debería haber incluido una antraciclina y un taxano.

Las reacciones adversas más comunes observadas durante el desarrollo de la molécula fueron neutropenia, leucopenia, astenia, fatiga, nausea, estreñimiento, alopecia, artralgia, mialgia, pirexia y neuropatía periférica.

\section{FINGOLIMOD $\left(\text { Gilenya }^{\circledR}\right)^{5}$}

Fingolimod se metaboliza a fosfato de fingolimod (metabolito activo), un antagonista funcional de los receptores de la esfingosina 1-fosfato (S1P) de linfocitos que redistribuye los linfocitos reduciendo la infiltración de linfocitos patógenos en el sistema nervioso central.

En los ensayos clínicos ha reducido el número de recaídas en pacientes con esclerosis múltiple remitente recidivante. $\mathrm{Su}$ indicación aprobada en monoterapia, es el tratamiento modificador para la esclerosis múltiple remitente-recidivante con elevada actividad de la enfermedad con beta-interferón así como para pacientes con esclerosis múltiple remitente-recidivante con evolución rápida de su gravedad definida esta por dos o más recaídas incapacitantes en un año, y con una o más lesiones cerebrales observadas con Gadolinio en resonancia magnética o un incremento significativo en el número y volumen de las lesiones en T2 de la resonancia magnética comparado con resonancias previas.

Las reacciones adversas más frecuentes durante la investigación clínica precomercialización fueron las infecciones por el virus de la gripe, dolor de cabeza, diarrea, dolor de espalda, tos y elevación de las enzimas hepáticas. Otras reacciones adversas comunes que podrían ser graves son la infección por el virus del herpes, linfopenia, leucopenia, bradicardia, bloqueo auriculoventricular, bronquitis $\mathrm{y}$ gastroenteritis.

\section{INHIBIDOR C1 HUMANO (Cinryze $\left.{ }^{\circledR}\right)^{1,7}$}

Este fármaco es un inhibidor de proteinasa que administrado por vía intravenosa aumenta los niveles funcionales del inhibidor $\mathrm{C} 1$ en pacientes con déficit de este, evitando la inflamación del angioedema hereditario.

La indicación aprobada es el tratamiento y prevención de los ataques de angioedema en adultos y adolescentes con angioedema hereditario. Prevención de los ataques de angioedema en adultos y adolescentes con ataques graves y recurrentes de angioedema hereditario, que sean intolerantes o que esten insuficientemente protegidos por tratamientos preventivos orales así como aquellos pacientes inadecuadamente controlados con tratamientos agudos repetidos.

La reacción adversa más frecuente observada durante el desarrollo clínico fue el rash.

\section{INMUNOGLOBULINA HUMANA NORMAL $\left(\text { Hizentra }^{\circledR}\right)^{5,8}$}

El principio activo es una inmunoglobulina humana normal que contiene principalmente Inmunoglobulina $\mathrm{G}$ (IgG) con un amplio espectro de anticuerpos frente a agentes infecciosos. Dosis adecuadas de este medicamento pueden restablecer niveles de IgG anormalmente bajos.

Las indicaciones aprobadas para Hizentra ${ }^{\circledR}$ son la terapia de sustitución en niños y adultos con síndrome de inmunodeficiencia primaria: agammaglobulinemia e hipogammaglobulinemia congénitas, inmunodeficiencia variable común, inmunodeficiencia combinada grave o deficiencias de subclases $\mathrm{IgG}$ con infecciones recurrentes y la terapia de sustitución en mieloma o en leucemia linfática crónica con hipogammaglobulinemia secundaria grave e infecciones recurrentes.

En un estudio llevado a cabo en Europa, fueron tratados con esta inmunoglobulina, durante más de 41 semanas, un total de 51 pacientes con síndromes de inmunodeficiencia primaria y con edades comprendidas entre 3 y 60 años. La dosis media administrada cada semana fue de $0,12 \mathrm{~g} / \mathrm{kg}$ peso. Durante el periodo de tratamiento se lograron unos niveles mantenidos de IgG entre 7,99-8,25 g/l. En otro ensayo clínico llevado a cabo en EE.UU., se trataron con Hizentra $^{\circledR}$, durante más de 15 meses, un total de 49 pacientes con síndromes de inmunodeficiencia primaria y con edades comprendidas entre 5 y 72 años. La dosis media administrada cada semana fue de $0,23 \mathrm{~g} / \mathrm{kg}$ peso. Durante el periodo de tratamiento se lograron unos niveles mantenidos de $\operatorname{IgG}$ de $12,53 \mathrm{~g} / \mathrm{l}$. No se comunicaron infecciones bacterianas durante el periodo de eficacia en los pacientes tratados con Hizentra ${ }^{\circledR}$ durante los estudios clínicos.

La seguridad del medicamento ha sido evaluada en varios estudios preclínicos con especial referencia al excipiente L-prolina. Los datos preclínicos no revelaron riesgos especiales para los humanos teniendo en consideración los estudios de seguridad farmacológica y de toxicidad.

\section{RETIGABINA $\left(\text { Trobalt }^{\circledR}\right)^{5}$}

La retigabina, es un antiepiléptico que activa específicamente los canales de potasio dependientes de voltaje KCNQ2-5 y su indicación aprobada es el tratamiento adyuvante de epilepsia en pacientes adultos mayores de 18 años en crisis de inicio parcial con o sin generalización secundaria.

Las reacciones adversas más comunes observadas durante el desarrollo clínico fueron reacciones adversas relacionadas con el $\mathrm{SNC}$, síntomas renales y del tracto urinario y efectos cardíacos.

\section{TAPENTADOL $\left(\right.$ Palexia $^{\circledR},{\left.\text { Palexia } \text { retard }^{\circledR}\right)^{5}}^{5}$}

Tapentadol es un analgésico potente con propiedades opioides agonistas del receptor $\mu$ y propiedades adicionales de inhibición de la recaptación de la noradrenalina.

Palexia ${ }^{\circledR}$ está indicado para el alivio del dolor agudo moderado a intenso en adultos, que sólo se puede tratar adecuadamente con un analgésico opioide mientras que Palexia retard ${ }^{\circledR}$ está indicado para controlar el dolor crónico intenso en adultos, que sólo se puede tratar adecuadamente con un analgésico opioide. 


\section{Evaluación positiva de medicamentos: 1 . $^{\text {er }}$ trimestre 2011}

No se ha establecido todavía la seguridad y eficacia en niños y adolescentes de menos de 18 años de edad. Por consiguiente, no se recomienda su uso en esta población.

Las reacciones adversas más frecuentes observadas durante el desarrollo clínico fueron gastrointestinales y del sistema nervioso central (náuseas, vómitos, somnolencia, mareos y cefalea), además tiene potencial de abuso y adicción. Hay que vigilar estrechamente a todos los pacientes tratados con principios activos que tienen actividad agonista de los receptores opioides $\mu$ para poder detectar los posibles signos de abuso y adicción.

\section{BIBLIOGRAFÍA}

1. Agencia Española de Medicamentos y Productos Sanitarios. Informe mensual sobre Medicamentos de Uso Humano y Productos Sanitarios Marzo 2011. Disponible en: http://www.aemps.es/actividad/notaMensual/2011/marzo2011/nota medicamentos.htm

2. Committee for medicinal products for human use (CHMP). Eliquis EMA CHMP/87152/2011. Disponible en: http://www.ema.europa.eu/docs/en_GB/document library/Summary of opinion - Initial authorisation/human/002148/ WC500103875.pdf
3. Committee for medicinal products for human use (CHMP). Yellox EMA/ CHMP/204341/2011. Disponible en: http://www.ema.europa.eu/docs/en GB/ document library/Summary of opinion - Initial authorisation/human/001198/ WC500103876.pdf

4. Agencia Española de Medicamentos y Productos Sanitarios. Informe mensual sobre Medicamentos de Uso Humano y Productos sanitarios Enero 2011. Disponible en: http://www.aemps.es/actividad/notaMensual/2011/enero2011/nota_medicamentos.htm

5. Agencia Española de Medicamentos y Productos Sanitarios. Informe mensual sobre Medicamentos de Uso Humano y Productos sanitarios Febrero 2011. Disponible en: http://www.aemps.es/actividad/notaMensual/2011/febrero2011/ nota_medicamentos.htm

6. Committee for medical products for human use (CHMP). Methylthioninium chloride Proveblue EMA/CHMP/82326/2011. Disponible en: http://www.ema. europa.eu/docs/en_GB/document_library/Summary_of_opinion_-_Initial_authorisation/human/002108/WC500102167.pdf

7. Committee for medical products for human use (CHMP). Cinryze EMA/ CHMP/217963/2011. Disponible en: http://www.ema.europa.eu/docs/en_GB/ document_library/Summary_of_opinion___Initial_authorisation/human/001207/ WC500103884.pdf

8. Committee for medical products for human use (CHMP). Hizentra EMA/ CHMP/110226/2011. Disponible en: http://www.ema.europa.eu/docs/en_GB/ document library/Summary of opinion - Initial authorisation/human/002127/ WC500102191.pdf 